\title{
NUMERICAL SOLUTION OF A MODEL FOR TURBULENT DIFFUSION
}

\author{
ERCILIA SOUSA \\ CMUC, Department of Mathematics, \\ University of Coimbra, 3001-454 Coimbra, Portugal \\ ecs@mat.uc.pt
}

Received December 7, 2012; Revised July 3, 2013

\begin{abstract}
A model is considered for turbulent diffusion which consists of a Riesz space fractional derivative to describe the turbulent phenomenon and also includes advection and classical diffusion. We present a first order explicit numerical method and a second order implicit numerical method to solve our problem and prove convergence results for both methods, including the derivation of stability constraints needed for the explicit numerical method to converge. In the end, to give some insights into the phenomenon of turbulent diffusion described by the Riesz fractional derivative, we show the behavior of the solution when we consider a Gaussian initial condition.
\end{abstract}

Keywords: Turbulent diffusion; fractional differential equations; finite differences; Riesz fractional derivative.

\section{The Model Problem}

Turbulence is difficult to define exactly, but there are several important characteristics that turbulent flows possess. These characteristics include unpredictability, rapid diffusivity and dissipation of kinetic energy.

Turbulent diffusion is a complex process that is very efficient at mixing pollutants in the natural environment thereby reducing the concentrations of potentially harmful contaminants to safe levels [Madja \& Kramer, 1999; Roberts \& Webster, 2002]. Despite many years of research, it is still poorly understood, and can only be rather crudely predicted in many cases. Demands for more reliable predictions, and predictions of quantities that have received little attention in the past are now increasing. These demands are also driven by the increase of applications of diffusion theory in new areas, where there is the need to quantify and predict, for example, instantaneous peak concentrations, intermittency of concentration fluctuations, the durations of concentration bursts and many other characteristics. Additionally, in several areas of diffusion research, there has been a rapid development of instrumentation techniques in the laboratory and the field. These have improved our ability to measure concentration fields enormously over the past ten years. The challenge now is to incorporate these new data into improved understanding and improved mathematical models of turbulent diffusion. The nonlocal nature of relative diffusion has stimulated the search for transport equations that differ significantly from conventional diffusive description.

A form of a diffusion equation that reflects the nonlocal character of transport and at the same time has a close relation to the conventional diffusion equation is given by [Bakunin, 2004, 2008]

$$
\frac{\partial u}{\partial t}(x, t)=\int_{-\infty}^{+\infty} G(x-\xi) u(\xi, t) d \xi,
$$

for a kernel function $G(x)$. The Fourier representation of the diffusion equation (1) is of the form

$$
\frac{\partial \hat{u}(k, t)}{\partial t}=\hat{G}(k) \hat{u}(k, t)
$$


which indicates the absence of memory effects for the Fourier harmonics. Here, we assume the function $u$ is defined in $\mathbb{R}$ and $\hat{G}(k)$ and $\hat{u}(k, t)$ are the Fourier transforms of the functions $G(x)$ and $u(x, t)$ with respect to the variable $x$.

If $\hat{G}(k)=-D k^{2}$ the Fourier representation corresponds to the classical diffusion equation, where $D$ is the conventional diffusion coefficient. However, the kernel function describing turbulent diffusion is given by [Bakunin, 2004; Chen, 2006; del-CastilloNegrete et al., 2005]

$$
\hat{G}(k)=-\epsilon^{1 / 3}|k|^{2 / 3},
$$

where $\epsilon$ is the mean energy dissipation rate. This representation is consistent with the approximation derived by Richardson under the assumption [Chukbar, 1993] that

$$
\hat{G}(k)=-D(k) k^{2}
$$

where the dependence $D(k)$ is given by [Boffetta \& Sokolov, 2002]

$$
D(k)=\epsilon^{1 / 3}|k|^{-4 / 3} .
$$

Using the fact that the Fourier transform of the function $\hat{G}$, can be interpreted in terms of fractional derivatives that have been used lately to describe nonlocality effects, an approximate solution of a problem describing turbulence is derived. The relation of this approach to the Lévy dynamics can be seen in [Shlesinger et al., 1987; Shlesinger et al., 1993] and the relation between Lévy dynamics and fractional derivatives is discussed, for instance, in [Zaslavsky, 2002].

We have the diffusion equation in Fourier representation given by

$$
\frac{\partial \hat{u}(k, t)}{\partial t}=-\epsilon^{1 / 3}|k|^{2 / 3} \hat{u}(k, t)
$$

Now, if we apply the inverse Fourier transform we have

$$
\frac{\partial u(x, t)}{\partial t}=\epsilon^{1 / 3} \mathcal{F}^{-1}\left[-|k|^{2 / 3} \hat{u}(k, t)\right] .
$$

Let us define a symmetric fractional derivative, through an inverse Fourier transform, that is,

$$
\frac{\partial^{\gamma} u}{\partial|x|^{\gamma}}(x, t)=\mathcal{F}^{-1}\left[-|k|^{\gamma} \hat{u}(k, t)\right],
$$

for $\gamma>0$, that can be defined for a sufficiently wellbehaved function $u(x, t), x \in \mathbb{R}$. Therefore, from Eq. (4) we obtain

$$
\frac{\partial u(x, t)}{\partial t}=\epsilon^{1 / 3} \frac{\partial^{2 / 3} u}{\partial|x|^{2 / 3}}(x, t) .
$$

To discuss the form of the symmetric fractional derivative let us recall the usual way of representing the fractional derivative which is given by the Riemann-Liouville formula.

The Riemann-Liouville fractional derivatives of order $\gamma$, for $n-1<\gamma \leq n$, of a function $u(x, t)$, for $x \in[a, b],-\infty \leq a<b \leq \infty$, are defined by [Podlubny, 1999]

$$
\begin{gathered}
\frac{\partial^{\gamma} u}{\partial x^{\gamma}}(x, t)=\frac{1}{\Gamma(n-\gamma)} \frac{\partial^{n}}{\partial x^{n}} \int_{a}^{x} \frac{u(\xi, t)}{(x-\xi)^{\gamma-n+1}} d \xi \\
n=[\gamma]+1, \quad x>a, \\
\frac{\partial^{\gamma} u}{\partial(-x)^{\gamma}}(x, t)=\frac{(-1)^{n}}{\Gamma(n-\gamma)} \frac{\partial^{n}}{\partial x^{n}} \int_{x}^{b} \frac{u(\xi, t)}{(\xi-x)^{\gamma-n+1}} d \xi \\
n=[\gamma]+1, \quad x<b,
\end{gathered}
$$

where $\Gamma(\cdot)$ is the Gamma function and $[a]$ denotes the integer part of $a$. Another way to represent the fractional derivatives is by the Grünwald-Letnikov formula, for $\gamma>0$, that is,

$$
\begin{aligned}
\frac{\partial^{\gamma} u}{\partial x^{\gamma}}(x, t)= & \lim _{\Delta x \rightarrow 0} \frac{1}{\Delta x^{\gamma}} \sum_{k=0}^{\left[\frac{x-a}{\Delta x}\right]}(-1)^{k} \\
& \times\left(\begin{array}{l}
\gamma \\
k
\end{array}\right) u(x-k \Delta x, t), \\
\frac{\partial^{\gamma} u}{\partial(-x)^{\gamma}}(x, t)= & \lim _{\Delta x \rightarrow 0} \frac{1}{\Delta x^{\gamma}} \sum_{k=0}^{\left[\frac{b-x}{\Delta x}\right]}(-1)^{k} \\
& \times\left(\begin{array}{l}
\gamma \\
k
\end{array}\right) u(x+k \Delta x, t) .
\end{aligned}
$$

In our particular case, these definitions will be equivalent, since we assume the function $u$ is defined in the whole real line, it is regular enough and verifies $\lim _{|x| \rightarrow \infty} u(x, t)=0$.

Now, we define the symmetric fractional derivative for $0<\gamma<1$. Let us first note that the Fourier transform of the left and right derivatives are given by

$$
\begin{aligned}
\mathcal{F}\left[\frac{\partial^{\gamma} u}{\partial x^{\gamma}}(x, t)\right] & =(-i k)^{\gamma} \hat{u}(x, t), \\
\mathcal{F}\left[\frac{\partial^{\gamma} u}{\partial(-x)^{\gamma}}(x, t)\right] & =(i k)^{\gamma} \hat{u}(x, t),
\end{aligned}
$$


and since

$$
\begin{gathered}
(-i k)^{\gamma}=|k|^{\gamma} \mathrm{e}^{-i \gamma \pi \operatorname{sgn}(k) / 2}, \\
(i k)^{\gamma}=|k|^{\gamma} \mathrm{e}^{i \gamma \pi \operatorname{sgn}(k) / 2}
\end{gathered}
$$

then

$$
\begin{aligned}
& -|k|^{\gamma} \hat{u}(k, t) \\
& =-\frac{1}{2 \cos \left(\frac{\gamma \pi}{2}\right)}\left[(-i k)^{\gamma} \hat{u}(k, t)+(i k)^{\gamma} \hat{u}(k, t)\right] .
\end{aligned}
$$

We can conclude that

$$
\begin{aligned}
\mathcal{F}^{-1}\left[-|k|^{\gamma} \hat{u}(k, t)\right] & \\
& =-\frac{1}{2 \cos \left(\frac{\gamma \pi}{2}\right)}\left[\frac{\partial^{\gamma} u}{\partial x^{\gamma}}(x, t)+\frac{\partial^{\gamma} u}{\partial(-x)^{\gamma}}(x, t)\right] .
\end{aligned}
$$

Therefore, the symmetric fractional space derivative can be written in terms of the left and right Riemann-Liouville derivatives as,

$$
\begin{aligned}
\frac{\partial^{\gamma} u}{\partial|x|^{\gamma}}(x, t)= & -\frac{1}{2 \cos \left(\frac{\pi \gamma}{2}\right)} \\
& \times\left[\frac{\partial^{\gamma} u}{\partial x^{\gamma}}(x, t)+\frac{\partial^{\gamma} u}{\partial(-x)^{\gamma}}(x, t)\right], \quad \gamma \neq 1 .
\end{aligned}
$$

This is called the Riesz derivative [Ortigueira, 2006; Samko et al., 1993; Zaslavsky, 2002].

If additionally to the anomalous diffusion in turbulence we want to have models that reflect intermittency, we need to add classical diffusion [Chen, 2006; Porta et al., 2001], that is,

$$
\frac{\partial u}{\partial t}(x, t)=d_{\gamma} \frac{\partial^{\gamma} u}{\partial|x|^{\gamma}}(x, t)+d_{2} \frac{\partial^{2} u}{\partial x^{2}}(x, t) .
$$

A survey on the existence of work on intermittency can be seen in [Sreenivasan \& Antonia, 1997]. To include the transport of fluids we consider the more general model with a constant velocity field $V$ [Chen \& Deem, 2001; Madja \& Kramer, 1999], that is,

$$
\begin{aligned}
\frac{\partial u}{\partial t}(x, t)= & -V \frac{\partial u}{\partial x}(x, t)+d_{\gamma} \frac{\partial^{\gamma} u}{\partial|x|^{\gamma}}(x, t) \\
& +d_{2} \frac{\partial^{2} u}{\partial x^{2}}(x, t) .
\end{aligned}
$$

As discussed in [Cushman-Roisin, 2008] turbulent diffusion can be seen as random advection. Note also that for this equation, the kernel function in (2) is given by

$$
\hat{G}(k)=\left(i V k-d_{\gamma}|k|^{\gamma}-d_{2} k^{2}\right) \hat{u}(k, t) .
$$

Instead of analyzing only the important case $\gamma=2 / 3$, we will consider the more general case $0<\gamma<1$.

\section{Approximate Solutions}

Let us consider the equation in the form

$$
\begin{aligned}
\frac{\partial u}{\partial t}(x, t)= & -V \frac{\partial u}{\partial x}(x, t)+d_{\gamma} \frac{\partial^{\gamma} u}{\partial|x|^{\gamma}}(x, t) \\
& +d_{2} \frac{\partial^{2} u}{\partial x^{2}}(x, t),
\end{aligned}
$$

where $V, d_{\gamma}, d_{2}>0$ and in general we assume $0<\gamma<1$ and in particular we consider $\gamma=2 / 3$. The problem is defined in the domain $\mathbb{R}$ with an initial condition

$$
u(x, 0)=f(x), \quad x \in \mathbb{R}
$$

and boundary conditions

$$
\lim _{x \rightarrow-\infty} u(x, t)=0 \quad \text { and } \quad \lim _{x \rightarrow \infty} u(x, t)=0 .
$$

In this section we develop numerical methods for the problem (14)-(16). To derive the numerical methods we suppose there are approximations $\mathbf{U}^{n}:=\left\{U_{j}^{n}\right\}$ to the values $U\left(x_{j}, t_{n}\right)$ at the mesh points

$$
x_{j}=j \Delta x, \quad j \in \mathbb{Z} \quad \text { and } \quad t_{n}=n \Delta t, n \geq 0,
$$

where $\Delta x$ denotes the uniform space step and $\Delta t$ the uniform time step. In the next sections we derive a first order explicit numerical method and a second order implicit numerical method. The first order numerical method considers the well known first order discretization derived from the GrünwaldLetnikov formula. The second order implicit numerical method uses a new second order discretization, derived following similar ideas to the ones presented in [Diethelm et al., 2004; Li et al., 2011; Sousa, 2012a, 2012b]. Additionally, we discuss the order of convergence of the new discretization and present some results on the stability of both numerical methods.

\subsection{A first order explicit method}

Discrete approximations to the fractional derivatives are defined from the Grünwald-Letnikov 
formulae, for all $\gamma>0$, respectively as

$$
\begin{array}{r}
\left(\frac{\partial^{\gamma} u}{\partial x^{\gamma}}\right)_{j}^{n}=\frac{1}{\Delta x^{\gamma}} \sum_{k=0}^{\infty} g_{k} U_{j-k}^{n}+\mathcal{O}(\Delta x), \\
\left(\frac{\partial^{\gamma} u}{\partial(-x)^{\gamma}}\right)_{j}^{n}=\frac{1}{\Delta x^{\gamma}} \sum_{k=0}^{\infty} g_{k} U_{j+k}^{n}+\mathcal{O}(\Delta x),
\end{array}
$$

where

$$
\begin{aligned}
g_{k} & =(-1)^{k}\left(\begin{array}{l}
\gamma \\
k
\end{array}\right) \\
& =(-1)^{k} \frac{\gamma(\gamma-1) \cdots(\gamma-k+1)}{k !} \\
& =\frac{\Gamma(k-\gamma)}{\Gamma(-\gamma) \Gamma(k+1)} .
\end{aligned}
$$

This approach is first order accurate as discussed in [Lubich, 1986; Podlubny, 1999; Li \& Zeng, 2012].

We plot, in Fig. 1, the coefficients $g_{k}$ for $\gamma=$ $2 / 3$. We observe that $g_{0}=1$ and $g_{k} \leq 0$ for all $k \geq 1$. Additionally we have that $\left|g_{k+1}\right| \leq\left|g_{k}\right|$, for all $k$. Note that, since the coefficients $g_{k}$ verify

$$
(1-z)^{\gamma}=\sum_{k=0}^{\infty} g_{k} z^{k}
$$

we have

$$
\sum_{k=0}^{\infty} g_{k}(-1)^{k}=2^{\gamma} \text { and } \quad \sum_{k=0}^{\infty} g_{k}=0
$$

Let us define

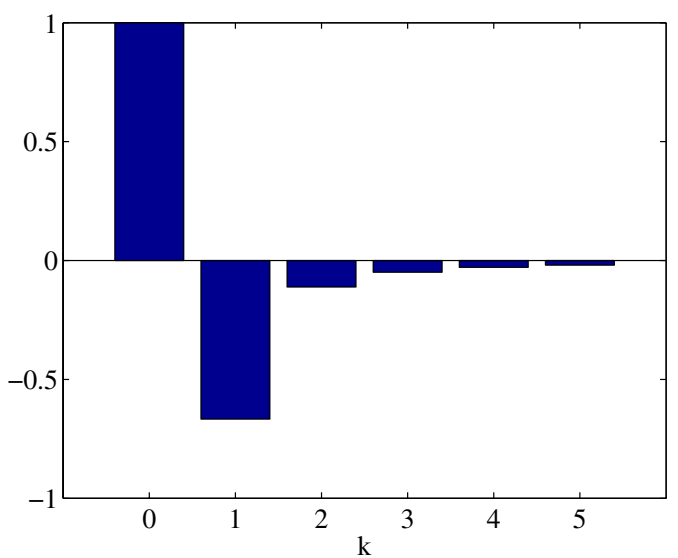

$$
\mu_{\gamma}=d_{\gamma} \frac{\Delta t}{\Delta x^{\gamma}}, \quad \mu_{2}=d_{2} \frac{\Delta t}{\Delta x^{2}} \quad \text { and } \quad \nu=V \frac{\Delta t}{\Delta x},
$$

and the differential operators

$$
\begin{aligned}
\delta^{2} U_{j}^{n} & =U_{j+1}^{n}-2 U_{j}^{n}+U_{j-1}^{n}, \\
\Delta_{-} U_{j}^{n} & =U_{j}^{n}-U_{j-1}^{n}
\end{aligned}
$$

and

$$
\Delta^{\gamma} U_{j}^{n}=-\frac{1}{2 \cos \left(\frac{\gamma \pi}{2}\right)}\left[\sum_{k=0}^{\infty} g_{k} U_{j-k}^{n}+\sum_{k=0}^{\infty} g_{k} U_{j+k}^{n}\right] .
$$

An explicit finite difference approximation for the turbulent diffusion equation (14) is given by

$$
U_{j}^{n+1}=U_{j}^{n}-\nu \Delta_{-} U_{j}^{n}+\mu_{\gamma} \Delta^{\gamma} U_{j}^{n}+\mu_{2} \delta^{2} U_{j}^{n} .
$$

\subsection{A second order implicit method}

We now derive a second order approximation for the left and right fractional derivatives in order to obtain in the end of the section an implicit second order numerical method. Consider first the left derivative, that is,

$$
\begin{gathered}
\frac{\partial^{\gamma} u}{\partial x^{\gamma}}(x, t)=\frac{1}{\Gamma(1-\gamma)} \frac{\partial}{\partial x} \int_{-\infty}^{x} u(\xi, t)(x-\xi)^{-\gamma} d \xi \\
0<\gamma<1
\end{gathered}
$$

We consider the discretization domain $x_{j}=j \Delta x$, $j \in \mathbb{Z}$. Let

$$
\mathcal{I}_{\gamma}^{l}(x)=\frac{1}{\Gamma(1-\gamma)} \int_{-\infty}^{x} u(\xi, t)(x-\xi)^{-\gamma} d \xi
$$

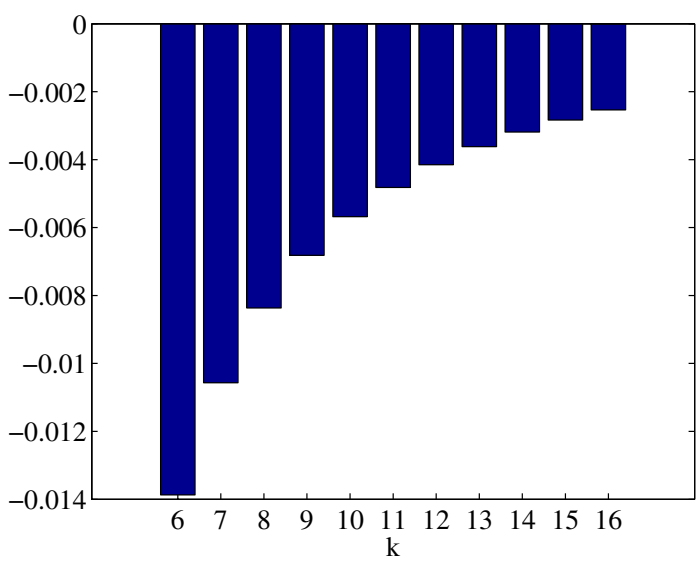

Fig. 1. Coefficients $g_{k}$ for $\gamma=2 / 3$. We have $g_{0}=1 ; g_{1}=-\gamma ; g_{2}=\gamma(\gamma-1) / 2 ; g_{k} \leq 0$, for all $k \geq 1$. 
First, the following approximation at $x_{j}$ is done,

$$
\frac{d}{d x} \mathcal{I}_{\gamma}^{l}\left(x_{j}\right) \simeq \frac{1}{2 \Delta x}\left[\mathcal{I}_{\gamma}^{l}\left(x_{j+1}\right)-\mathcal{I}_{\gamma}^{l}\left(x_{j-1}\right)\right] .
$$

Secondly, we compute these integrals by approximating the function $u$ by a linear spline $s_{j}(\xi)$, whose nodes and knots are chosen at $x_{k}, k \leq j$. Similar approaches have been done in [Diethelm et al., 2004; Li et al., 2011; Sousa, 2012a, 2012b]. Therefore, an approximation to (24) becomes

$$
I_{\gamma}^{l}\left(x_{j}\right)=\frac{1}{\Gamma(1-\gamma)} \int_{-\infty}^{x_{j}} s_{j}(\xi)\left(x_{j}-\xi\right)^{-\gamma} d \xi
$$

where the spline $s_{j}(\xi)$ interpolates $\left\{u\left(x_{k}, t\right): k \leq j\right\}$ in the interval $(-\infty, x]$ and is of the form

$$
s_{j}(\xi)=\sum_{k=-\infty}^{j} u\left(x_{k}, t\right) s_{j, k}(\xi),
$$

with $s_{j, k}(\xi)$, in each interval $\left[x_{k-1}, x_{k+1}\right]$, for $k \leq$ $j-1$, given by

$$
s_{j, k}(\xi)= \begin{cases}\frac{\xi-x_{k-1}}{x_{k}-x_{k-1}}, & x_{k-1} \leq \xi \leq x_{k} \\ \frac{x_{k+1}-\xi}{x_{k+1}-x_{k}}, & x_{k} \leq \xi \leq x_{k+1} \\ 0 & \text { otherwise }\end{cases}
$$

and for $k=j$ in the interval $\left[x_{j-1}, x_{j}\right], s_{j, k}(\xi)$ is of the form

$$
s_{j, j}(\xi)= \begin{cases}\frac{\xi-x_{j-1}}{x_{j}-x_{j-1}}, & x_{j-1} \leq \xi \leq x_{j} \\ 0 & \text { otherwise. }\end{cases}
$$

We obtain,

$$
\begin{aligned}
I_{\gamma}^{l}\left(x_{j}\right)= & \frac{1}{(2-\gamma)(1-\gamma) \Gamma(1-\gamma)} \Delta x^{1-\gamma} \\
& \times \sum_{k=-\infty}^{j} u\left(x_{k}, t\right) a_{j, k},
\end{aligned}
$$

where the $a_{j, k}$ are defined by

$$
a_{j, k}=\left\{\begin{array}{cl}
(j-k+1)^{2-\gamma}-2(j-k)^{2-\gamma} & \\
+(j-k-1)^{2-\gamma}, & k \leq j-1 \\
1, & k=j .
\end{array}\right.
$$

Therefore, we have

$$
\begin{aligned}
\frac{d}{d x} \mathcal{I}_{\gamma}^{l}\left(x_{j}\right) \simeq & \frac{1}{2 \Delta x}\left[I_{\gamma}^{l}\left(x_{j+1}\right)-I_{\gamma}^{l}\left(x_{j-1}\right)\right] \\
= & \frac{\Delta x^{1-\gamma}}{2 \Delta x \Gamma(3-\gamma)}\left[\sum_{k=-\infty}^{j+1} u\left(x_{k}, t\right) a_{j+1, k}\right. \\
& \left.-\sum_{k=-\infty}^{j-1} u\left(x_{k}, t\right) a_{j-1, k}\right] .
\end{aligned}
$$

Finally, an approximation to (23) is given by $\frac{\delta_{l}^{\gamma} u\left(x_{j}, t\right)}{\Delta x^{\gamma}}$, where

$$
\delta_{l}^{\gamma} u\left(x_{j}, t\right)=\frac{1}{2 \Gamma(3-\gamma)} \sum_{k=-\infty}^{j+1} b_{j, k} u\left(x_{k}, t\right),
$$

for

$$
\begin{aligned}
b_{j, k} & =a_{j+1, k}-a_{j-1, k}, \quad k \leq j-1 \\
b_{j, j} & =a_{j+1, j} \\
b_{j, j+1} & =a_{j+1, j+1} .
\end{aligned}
$$

Let us rewrite the previous fractional operator. For that, let us define,

$$
a_{m}= \begin{cases}(m+1)^{2-\gamma}-2 m^{2-\gamma}+(m-1)^{2-\gamma}, & m \geq 1 \\ 1, & m=0\end{cases}
$$

and

$$
b_{m}= \begin{cases}a_{m+1}-a_{m-1}, & m \geq 1 \\ a_{1}, & m=0 \\ a_{0}, & m=-1 .\end{cases}
$$

We can rewrite the fractional operator in the following way,

$$
\delta_{l}^{\gamma} u\left(x_{j}, t\right)=\frac{1}{2 \Gamma(3-\gamma)} \sum_{m=-1}^{\infty} b_{m} u\left(x_{j-m}, t\right) .
$$

Similarly we can derive the right Riemann-Liouville derivative approximation to obtain,

$$
\delta_{r}^{\gamma} u\left(x_{j}, t\right)=\frac{1}{2 \Gamma(3-\gamma)} \sum_{m=-1}^{\infty} b_{m} u\left(x_{j+m}, t\right) .
$$

We denote

$$
\Delta_{2}^{\gamma} u\left(x_{j}, t\right)=-\frac{1}{2 \cos \left(\frac{\gamma \pi}{2}\right)}\left[\delta_{l}^{\gamma} u\left(x_{j}, t\right)+\delta_{r}^{\gamma} u\left(x_{j}, t\right)\right] .
$$



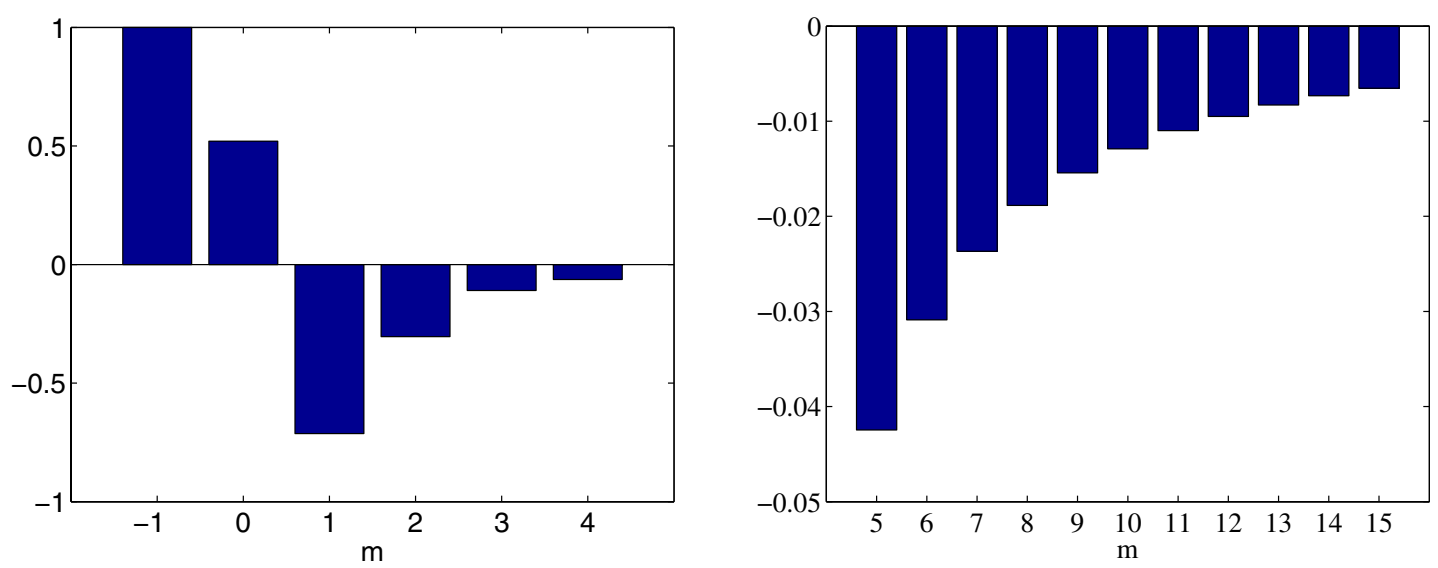

Fig. 2. Coefficients $b_{m}$ for $\gamma=2 / 3$. We have $b_{-1}=1 ; b_{0}=2^{2-\gamma}-2 ; b_{m} \leq 0$, for $m \geq 1$.

We plot, in Fig. 2, the coefficients $b_{m}$ for $\gamma=$ $2 / 3$. We observe that $b_{-1}=1, b_{0}=2^{2-\gamma}-2$ and $b_{m} \leq 0$ for all $m \geq 1$. Additionally we have that $\left|b_{m+1}\right| \leq\left|b_{m}\right|$, for all $m \geq 1$.

Let us define the central difference operator

$$
\Delta_{0} U_{j}^{n}=\frac{1}{2}\left(U_{j+1}^{n}-U_{j-1}^{n}\right)
$$

An implicit finite difference approximation for the turbulent diffusion equation is given by

$$
\begin{aligned}
& {\left[1-\frac{1}{2}\left(-\nu \Delta_{0}+\mu_{\gamma} \Delta_{2}^{\gamma}+\mu_{2} \delta^{2}\right)\right] U_{j}^{n+1}} \\
& \quad=\left[1+\frac{1}{2}\left(-\nu \Delta_{0}+\mu_{\gamma} \Delta_{2}^{\gamma}+\mu_{2} \delta^{2}\right)\right] U_{j}^{n}
\end{aligned}
$$

\subsection{Convergence analysis}

In order to discuss the convergence of the numerical methods we study their consistency and numerical stability. We start with the consistency, which guarantees that the difference formulas converge to the differential equation as $\Delta t$ and $\Delta x$ go to zero. Then we derive under which conditions the solution is numerically stable, that is, the conditions which guarantee the error stays bounded and does not grow to be much larger during calculations.

To state the consistency, we start to prove that the approximations given by (34) and (35) are second order accurate. We prove this result only for the approximation of the left Riemann-Liouville derivative (34) since it is then straightforward to conclude that a similar result is valid for the approximation of the right Riemann-Liouville derivative (35).
For a clear presentation, we omit the variable $t$ in the next lemma and theorem and we denote the partial derivative of $u$ in $x$ of order $r$ by $u^{(r)}$.

Lemma 1. Let $u \in C^{(3)}(\mathbb{R})$. For $\xi \in\left[x_{k-1}, x_{k}\right]$,

$$
\begin{array}{r}
u(\xi)-s_{j, k}(\xi)=-\frac{1}{2} u^{(2)}(\xi) l_{k, 2}(\xi)-\frac{1}{6} u^{(3)}\left(\eta_{k}\right) l_{k, r}(\xi), \\
\eta_{k} \in\left[x_{k-1}, x_{k}\right],
\end{array}
$$

where $\left|l_{k, r}(\xi)\right| \leq \Delta x^{r}, r=2,3$.

Proof. For $\xi \in\left[x_{k-1}, x_{k}\right]$,

$$
\begin{aligned}
u(\xi)-s_{j, k}(\xi)= & u(\xi)-\frac{x_{k}-\xi}{\Delta x} u\left(x_{k-1}\right) \\
& -\frac{\xi-x_{k-1}}{\Delta x} u\left(x_{k}\right) .
\end{aligned}
$$

Using Taylor expansions, we obtain

$u(\xi)-s_{j, k}(\xi)=-\frac{1}{2} u^{(2)}(\xi) l_{k, 2}(\xi)-\frac{1}{6} u^{(3)}\left(\eta_{k}\right) l_{k, 3}(\xi)$,

where $l_{k, r}(\xi)$ are functions which depend on $\Delta x$ and $x_{k}$, given by

$$
\begin{aligned}
l_{k, r}(\xi)= & \frac{x_{k}-\xi}{\Delta x}\left(x_{k}-\xi-\Delta x\right)^{r} \\
& -\frac{\xi-x_{k}+\Delta x}{\Delta x}\left(x_{k}-\xi\right)^{r} \\
= & \left(x_{k}-\xi\right)^{r}+\sum_{r=0}^{p-1}\left(\begin{array}{l}
r \\
p
\end{array}\right)\left(x_{k}-\xi\right)^{p+1} \\
& \times(-1)^{r-p} \Delta x^{r-p-1} .
\end{aligned}
$$


It is easy to conclude that $\left|l_{k, r}(\xi)\right| \leq \Delta x^{r}$, for $\xi \in\left[x_{k-1}, x_{k}\right]$.

Theorem 2 (Order of Accuracy of the Approximation for the Left Fractional Derivative). Let $u \in$ $C^{(3)}(\mathbb{R})$ and such that, the third-order derivative, $u^{(3)}$, has compact support. We have that

$$
\frac{\partial^{\gamma} u}{\partial x^{\gamma}}\left(x_{j}\right)-\frac{\delta_{l}^{\gamma} u}{\Delta x^{\gamma}}\left(x_{j}\right)=\epsilon_{1}\left(x_{j}\right)+\epsilon_{2}\left(x_{j}\right),
$$

where

$$
\left|\epsilon_{1}\left(x_{j}\right)\right| \leq C_{1} \Delta x^{2}, \quad\left|\epsilon_{2}\left(x_{j}\right)\right| \leq C_{2} \Delta x^{2},
$$

and $C_{1}$ and $C_{2}$ are independent of $\Delta x$.

Proof. We start to note that we have

$$
\begin{aligned}
\frac{\partial^{\gamma} u}{\partial x^{\gamma}}\left(x_{j}\right) & =\frac{\partial}{\partial x} \mathcal{I}_{\gamma}^{l}\left(x_{j}\right) \\
& =\frac{1}{2 \Delta x}\left[\mathcal{I}_{\gamma}^{l}\left(x_{j+1}\right)-\mathcal{I}_{\gamma}^{l}\left(x_{j-1}\right)\right]+\epsilon_{1}\left(x_{j}\right),
\end{aligned}
$$

where $\epsilon_{1}\left(x_{j}\right)=\mathcal{O}\left(\Delta x^{2}\right)$.

Let us now define the error $E\left(x_{j}\right)$, such that,

$$
\begin{aligned}
\mathcal{I}_{\gamma}^{l}\left(x_{j+1}\right)-\mathcal{I}_{\gamma}^{l}\left(x_{j-1}\right)= & I_{\gamma}^{l}\left(x_{j+1}\right)-I_{\gamma}^{l}\left(x_{j-1}\right) \\
& +\frac{1}{\Gamma(1-\gamma)} E\left(x_{j}\right),
\end{aligned}
$$

where $\mathcal{I}_{\gamma}^{l}$ and $I_{\gamma}^{l}$ are defined in (24) and (26) respectively. We have

$$
\begin{aligned}
\frac{\partial^{\gamma} u}{\partial x^{\gamma}}\left(x_{j}\right)= & \frac{1}{2 \Delta x}\left[I_{\gamma}^{l}\left(x_{j+1}\right)-I_{\gamma}^{l}\left(x_{j-1}\right)\right] \\
& +\frac{1}{\Gamma(1-\gamma)} \frac{1}{2 \Delta x} E\left(x_{j}\right)+\epsilon_{1}\left(x_{j}\right)
\end{aligned}
$$

that is

$$
\frac{\partial^{\gamma} u}{\partial x^{\gamma}}\left(x_{j}\right)=\frac{\delta_{l}^{\gamma} u}{\Delta x^{\gamma}}\left(x_{j}\right)+\epsilon_{1}\left(x_{j}\right)+\epsilon_{2}\left(x_{j}\right),
$$

where

$$
\epsilon_{2}\left(x_{j}\right)=\frac{1}{\Gamma(1-\gamma)} \frac{1}{2 \Delta x} E\left(x_{j}\right) .
$$

From $(39)$ the error $E\left(x_{j}\right)$ is given by

$$
\begin{aligned}
E\left(x_{j}\right)= & \sum_{k=-\infty}^{j+1} \int_{x_{k-1}}^{x_{k}}\left(u(\xi)-s_{j+1, k}(\xi)\right) \\
& \times\left(x_{j+1}-\xi\right)^{-\gamma} d \xi
\end{aligned}
$$

$$
\begin{aligned}
& -\sum_{k=-\infty}^{j-1} \int_{x_{k-1}}^{x_{k}}\left(u(\xi)-s_{j-1, k}(\xi)\right) \\
& \times\left(x_{j-1}-\xi\right)^{-\gamma} d \xi
\end{aligned}
$$

Taking in consideration the previous lemma, we can write $E\left(x_{j}\right)$ as,

$$
E\left(x_{j}\right)=-\frac{1}{2} E_{2}\left(x_{j}\right)-\frac{1}{6} E_{3}\left(x_{j}\right),
$$

where $E_{2}\left(x_{j}\right)$ and $E_{3}\left(x_{j}\right)$ are defined as follows,

$$
\begin{aligned}
E_{2}\left(x_{j}\right)= & \sum_{k=-\infty}^{j+1} \int_{x_{k-1}}^{x_{k}} l_{k, 2}(\xi) u^{(2)}(\xi)\left(x_{j+1}-\xi\right)^{-\gamma} d \xi \\
& -\sum_{k=-\infty}^{j-1} \int_{x_{k-1}}^{x_{k}} l_{k, 2}(\xi) u^{(2)}(\xi) \\
& \times\left(x_{j-1}-\xi\right)^{-\gamma} d \xi \\
E_{3}\left(x_{j}\right)= & \sum_{k=-\infty}^{j+1} u^{(3)}\left(\eta_{k}\right) \int_{x_{k-1}}^{x_{k}} l_{k, 3}(\xi)\left(x_{j+1}-\xi\right)^{-\gamma} d \xi \\
& +\sum_{k=-\infty}^{j-1} u^{(3)}\left(\eta_{k}\right) \int_{x_{k-1}}^{x_{k}} l_{k, 3}(\xi) \\
& \times\left(x_{j-1}-\xi\right)^{-\gamma} d \xi .
\end{aligned}
$$

For $E_{2}\left(x_{j}\right)$ by changing variables, we obtain

$$
\begin{aligned}
E_{2}\left(x_{j}\right)= & \sum_{k=-\infty}^{j} \int_{x_{k-1}}^{x_{k}} l_{k, 2}(\xi) u^{(2)}(\xi+\Delta x) \\
& \times\left(x_{j}-\xi\right)^{-\gamma} d \xi \\
& -\sum_{k=-\infty}^{j} \int_{x_{k-1}}^{x_{k}} l_{k, 2}(\xi) u^{(2)}(\xi-\Delta x) \\
& \times\left(x_{j}-\xi\right)^{-\gamma} d \xi
\end{aligned}
$$

that is,

$$
\begin{aligned}
E_{2}\left(x_{j}\right)= & \sum_{k=-\infty}^{j} \int_{x_{k-1}}^{x_{k}} l_{k, 2}(\xi) \\
& \times\left[u^{(2)}(\xi+\Delta x)-u^{(2)}(\xi-\Delta x)\right] \\
& \times\left(x_{j}-\xi\right)^{-\gamma} d \xi
\end{aligned}
$$


Since the third order derivative has compact support, there exists $x_{a}=N_{a} \Delta x$ such that $u^{(3)}(x)=0$, for $x \leq x_{a}$. Then, by Taylor expansions we obtain,

$$
\begin{aligned}
E_{2}\left(x_{j}\right)= & 2 \Delta x \sum_{k=N_{a}+1}^{j} \int_{x_{k-1}}^{x_{k}} l_{k, 2}(\xi) u^{(3)}\left(\xi_{k}\right) \\
& \times\left(x_{j}-\xi\right)^{-\gamma} d \xi, \quad \xi_{k} \in\left[x_{k-1}, x_{k}\right] .
\end{aligned}
$$

Then

$$
\left|E_{2}\left(x_{j}\right)\right| \leq 2 \Delta x\left\|u^{(3)}\right\|_{\infty} \sum_{k=N_{a}+1}^{j}\left|c_{j, k, 2}\right|,
$$

where

$$
c_{j, k, 2}=\int_{x_{k-1}}^{x_{k}} l_{k, 2}(\xi)\left(x_{j}-\xi\right)^{-\gamma} d \xi .
$$

Since, by Lemma 1 ,

$$
\left|c_{j, k, 2}\right| \leq \Delta x^{2} \int_{x_{k-1}}^{x_{k}}\left(x_{j}-\xi\right)^{-\gamma} d \xi
$$

and

$$
\int_{x_{a}}^{x_{j}}\left(x_{j}-\xi\right)^{-\gamma} d \xi=\frac{1}{1-\gamma}\left(x_{j}-x_{a}\right)^{1-\gamma}
$$

we have

$$
\left|E_{2}\left(x_{j}\right)\right| \leq \frac{2 \Delta x^{3}}{(1-\gamma)}\left\|u^{(3)}\right\|_{\infty}\left(x_{j}-x_{a}\right)^{1-\gamma} .
$$

For $E_{3}\left(x_{j}\right)$, we bound each integral of (43) separately. For the first integral we have

$$
\begin{aligned}
& \sum_{k=N_{a}+1}^{j+1} u^{(3)}\left(\eta_{k}\right) \int_{x_{k-1}}^{x_{k}} l_{k, 3}(\xi)\left(x_{j+1}-\xi\right)^{-\gamma} d \xi \\
& \leq \Delta x^{3}\left\|u^{(3)}\right\|_{\infty} \sum_{k=N_{a}+1}^{j+1} \int_{x_{k-1}}^{x_{k}}\left(x_{j+1}-\xi\right)^{-\gamma} d \xi \\
& \quad=\frac{\Delta x^{3}}{1-\gamma}\left\|u^{(3)}\right\|_{\infty}\left(x_{j+1}-x_{a}\right)^{1-\gamma} .
\end{aligned}
$$

Therefore, since $(a+b)^{p} \leq|a|^{p}+|b|^{p}$ for $0<p \leq 1$, we have

$$
\begin{aligned}
& \sum_{k=N_{a}+1}^{j+1} u^{(3)}\left(\eta_{k}\right) \int_{x_{k-1}}^{x_{k}} l_{k, 3}(\xi)\left(x_{j+1}-\xi\right)^{-\gamma} d \xi \\
& \quad \leq \frac{\Delta x^{3}}{1-\gamma}\left\|u^{(3)}\right\|_{\infty}\left(\left(x_{j}-x_{a}\right)^{1-\gamma}+\Delta x^{1-\gamma}\right) .
\end{aligned}
$$

Similarly, for the second integral

$$
\begin{aligned}
& \sum_{k=N_{a}+1}^{j-1} u^{(3)}\left(\eta_{k}\right) \int_{x_{k-1}}^{x_{k}} l_{k, 3}(\xi)\left(x_{j-1}-\xi\right)^{-\gamma} d \xi \\
& \quad \leq \frac{\Delta x^{3}}{1-\gamma}\left\|u^{(3)}\right\|_{\infty}\left(\left(x_{j}-x_{a}\right)^{1-\gamma}+\Delta x^{1-\gamma}\right) .
\end{aligned}
$$

Finally, we have

$$
\begin{aligned}
\left|E_{3}\left(x_{j}\right)\right| \leq & \frac{2 \Delta x^{3}}{1-\gamma}\left\|u^{(3)}\right\|_{\infty}\left(x_{j}-x_{a}\right)^{1-\gamma} \\
& +\frac{2 \Delta x^{4-\gamma}}{1-\gamma}\left\|u^{(3)}\right\|_{\infty} .
\end{aligned}
$$

From (44) and (45) it is easy to conclude that the error $E\left(x_{j}\right)$ defined by (41) is of order $\mathcal{O}\left(\Delta x^{3}\right)$ and therefore the $\epsilon_{2}\left(x_{j}\right)$ is of order $\mathcal{O}\left(\Delta x^{2}\right)$, for $0<\gamma<1$.

Remark. If we consider the fractional derivative of a function $u$ defined in $[a, b]$ and such that $u \in$ $C^{(3)}([a, b])$ with $u^{(r)}(a)=0$ for $r=0,1,2$, it is easy to conclude that the previous result still holds, since we can consider an extension $\tilde{u}$ to $\mathbb{R}$, such that $\tilde{u} \in C^{(3)}(\mathbb{R})$ with $\tilde{u}(x)=u(x)$, for $x \in[a, b]$ and zero otherwise.

Remark. For a function $u$ with derivatives until order two are not zero at the boundaries, let us analyze the additional error we would get theoretically, with this type of discretization. Consider the error $E\left(x_{j}\right)$ defined in (41) through the errors (42) and (43), for a bounded domain, where now $x_{j}=$ $a+j \Delta x$. We have,

$$
\begin{aligned}
E_{2}\left(x_{j}\right)= & \sum_{k=1}^{j+1} \int_{x_{k-1}}^{x_{k}} l_{k, 2}(\xi) u^{(2)}(\xi)\left(x_{j+1}-\xi\right)^{-\gamma} d \xi \\
& -\sum_{k=1}^{j-1} \int_{x_{k-1}}^{x_{k}} l_{k, 2}(\xi) u^{(2)}(\xi)\left(x_{j-1}-\xi\right)^{-\gamma} d \xi \\
= & \sum_{k=0}^{j} \int_{x_{k-1}}^{x_{k}} l_{k, 2}(\xi) u^{(2)}(\xi+\Delta x)\left(x_{j}-\xi\right)^{-\gamma} d \xi \\
& -\sum_{k=2}^{j} \int_{x_{k-1}}^{x_{k}} l_{k, 2}(\xi) u^{(2)}(\xi-\Delta x) \\
& \times\left(x_{j}-\xi\right)^{-\gamma} d \xi .
\end{aligned}
$$


Therefore,

$$
\begin{aligned}
E_{2}\left(x_{j}\right)= & \epsilon_{2}^{*}\left(x_{j}\right)+\sum_{k=2}^{j} \int_{x_{k-1}}^{x_{k}} l_{k, 2}(\xi)\left(u^{(2)}(\xi+\Delta x)\right. \\
& \left.-u^{(2)}(\xi-\Delta x)\right)\left(x_{j}-\xi\right)^{-\gamma} d \xi
\end{aligned}
$$

where

$\epsilon_{2}^{*}\left(x_{j}\right)=\sum_{k=0}^{1} \int_{x_{k-1}}^{x_{k}} l_{k, 2}(\xi) u^{(2)}(\xi+\Delta x)\left(x_{j}-\xi\right)^{-\gamma} d \xi$.

The different order of accuracy, for this case, can come from the error $\epsilon_{2}^{*}\left(x_{j}\right)$, since for the second term we can proceed similarly as in the previous theorem.

It is also easy to see that for the error $E_{3}\left(x_{j}\right)$ defined by (43) the analysis that was done in the previous theorem for $\mathbb{R}$ follows straightforward for a bounded interval. Therefore the only additional term we need to discuss is $\epsilon_{2}^{*}\left(x_{j}\right)$. We have that

$$
\begin{aligned}
& \left|\epsilon_{2}^{*}\left(x_{1}\right)\right| \leq \Delta x^{3-\gamma} \frac{2^{1-\gamma}}{1-\gamma} \max _{x_{0} \leq \xi \leq x_{2}}\left|u^{(2)}(\xi)\right| ; \\
& \left|\epsilon_{2}^{*}\left(x_{j}\right)\right| \leq \Delta x^{3-\gamma} \frac{2}{(j-1)^{\gamma}} \max _{x_{0} \leq \xi \leq x_{2}}\left|u^{(2)}(\xi)\right|,
\end{aligned}
$$$$
\text { for } j \geq 2 \text {. }
$$

Therefore, in addition to the orders of the errors stated in the previous theorem we would have an order related to this term of $\Delta x^{2-\gamma}$. Note that if $u^{(2)}$ is small enough near the boundary, we have second order accuracy. Additionally the order of accuracy will be very close to two for values of $\gamma$ close to 0 . Note also that for the discrete points faraway from the boundary the error $\epsilon_{2}^{*}\left(x_{j}\right)$ can be significantly smaller.

Now, the consistency comes straightforward from the fact that the approximation of the derivatives is consistent. Let $u=u(x, t)$ be the exact solution. The truncation errors for the explicit and implicit numerical methods are respectively given by

$$
\begin{aligned}
T_{E_{j}}^{n}= & \frac{u_{j}^{n+1}-u_{j}^{n}}{\Delta t}+V \frac{u_{j}^{n}-u_{j-1}^{n}}{\Delta x}-\frac{d_{\gamma}}{\Delta x^{\gamma}} \Delta^{\gamma} u_{j}^{n} \\
& -\frac{d_{2}}{\Delta x^{2}}\left(u_{j+1}^{n}-2 u_{j}^{n}-u_{j-1}^{n}\right) \\
= & \left(\frac{\partial u}{\partial t}\right)_{j}^{n}+\mathcal{O}(\Delta t)+V\left(\frac{\partial u}{\partial x}\right)_{j}^{n}+\mathcal{O}(\Delta x)
\end{aligned}
$$

$$
\begin{aligned}
& -d_{\gamma}\left(\frac{\partial^{\gamma} u}{\partial|x|^{\gamma}}\right)_{j}^{n}+\mathcal{O}(\Delta x) \\
& -d_{2}\left(\frac{\partial^{2} u}{\partial x^{2}}\right)_{j}^{n}+\mathcal{O}\left(\Delta x^{2}\right)
\end{aligned}
$$

and

$$
\begin{aligned}
T_{I j}^{n}= & \frac{u_{j}^{n+1}-u_{j}^{n}}{\Delta t}+\frac{1}{2} \frac{V}{\Delta x}\left(\Delta_{0} u_{j}^{n+1}-\Delta_{0} u_{j}^{n}\right) \\
& -\frac{1}{2}\left\{\frac{d_{\gamma}}{\Delta x^{\gamma}}\left(\Delta_{2}^{\gamma} u_{j}^{n+1}+\Delta_{2}^{\gamma} u_{j}^{n}\right)\right. \\
& \left.+\frac{d_{2}}{\Delta x^{2}}\left(\delta^{2} u_{j}^{n+1}+\delta^{2} u_{j}^{n}\right)\right\}
\end{aligned}
$$

We have

$$
\begin{aligned}
T_{I j}^{n}= & \left(\frac{\partial u}{\partial t}\right)_{j}^{n+1 / 2}+\mathcal{O}\left(\Delta t^{2}\right)+V\left(\frac{\partial u}{\partial x}\right)_{j}^{n+1 / 2} \\
& -d_{\gamma}\left(\frac{\partial^{\gamma} u}{\partial|x| \gamma}\right)_{j}^{n+1 / 2}-d_{2}\left(\frac{\partial^{2} u}{\partial x^{2}}\right)_{j}^{n+1 / 2} \\
& +\mathcal{O}\left(\Delta x^{2}\right)+\mathcal{O}\left(\Delta t^{2}\right)
\end{aligned}
$$

and therefore

$$
\begin{gathered}
T_{E_{j}}^{n}=\mathcal{O}(\Delta t)+\mathcal{O}(\Delta x), \\
T_{I_{j}}^{n}=\mathcal{O}\left(\Delta t^{2}\right)+\mathcal{O}\left(\Delta x^{2}\right) .
\end{gathered}
$$

In order to derive stability conditions for the finite difference schemes, we apply the von Neumann analysis. If $u_{j}^{n}$ is the exact solution $u\left(x_{j}, t_{n}\right)$, let $U_{j}^{n}$ be a perturbation of $u_{j}^{n}$. The perturbation error

$$
e_{j}^{n}=U_{j}^{n}-u_{j}^{n}
$$

will be propagated forward in time according to the equations respectively

$$
\begin{aligned}
& e_{j}^{n+1}=e_{j}^{n}-\nu \Delta_{-} e_{j}^{n}+\mu_{\gamma} \Delta^{\gamma} e_{j}^{n}+\mu_{2} \delta^{2} e_{j}^{n}, \\
& {\left[1-\frac{1}{2}\left(-\nu \Delta_{0}+\mu_{\gamma} \Delta_{2}^{\gamma}+\mu_{2} \delta^{2}\right)\right] e_{j}^{n+1}} \\
& \quad=\left[1+\frac{1}{2}\left(-\nu \Delta_{0}+\mu_{\gamma} \Delta_{2}^{\gamma}+\mu_{2} \delta^{2}\right)\right] e_{j}^{n} .
\end{aligned}
$$

The von Neumann analysis assumes that the error $e_{j}^{n}$ will be decomposed into a Fourier series with terms given by $\kappa_{p}^{n} e^{i \xi_{p}(j \Delta x)}$, where $\kappa_{p}^{n}$ is the amplitude of the $p$ th harmonic. The product $\xi_{p} \Delta x$ 
is the phase angle $\theta=\xi_{p} \Delta x$ and covers the domain $[-\pi, \pi]$.

Considering a single mode $\kappa^{n} e^{i j \theta}$, its time evolution is determined by the same numerical scheme as the error $e_{j}^{n}$. By inserting a representation of this form into a numerical scheme we obtain stability conditions. The stability conditions will be satisfied if the amplitude factor $\kappa$ does not grow in time, that is, if we have $|\kappa(\theta)| \leq 1$, for all $\theta$.

The next theorem concerns the explicit numerical method that, as expected, is conditionally stable.

Theorem 3. A necessary stability condition for the explicit numerical method (22) is

$$
\nu+2 \mu_{2}+\frac{1}{2^{1-\gamma} \cos \left(\frac{\pi \gamma}{2}\right)} \mu_{\gamma} \leq 1 .
$$

Proof. If we insert the mode $\kappa^{n} \mathrm{e}^{i j \theta}$ into (22) then

$$
\begin{aligned}
\kappa(\theta)= & 1-\nu\left(1-\mathrm{e}^{-i \theta}\right)+\mu_{2}\left(\mathrm{e}^{i \theta}-2+\mathrm{e}^{-i \theta}\right) \\
& -\frac{\mu_{\gamma}}{2 \cos \left(\frac{\gamma \pi}{2}\right)}\left[\sum_{k=0}^{\infty} g_{k} \mathrm{e}^{-i k \theta}+\sum_{k=0}^{\infty} g_{k} \mathrm{e}^{i k \theta}\right] .
\end{aligned}
$$

Consequently, considering the phase angle $\theta=\pi$,

$$
\begin{aligned}
\kappa(\pi) & =1-2 \nu-4 \mu_{2}-\frac{\mu_{\gamma}}{2 \cos \left(\frac{\gamma \pi}{2}\right)} \sum_{k=0}^{\infty} g_{k}(-1)^{k} \\
& =1-2 \nu-4 \mu_{2}-\frac{\mu_{\gamma}}{2 \cos \left(\frac{\gamma \pi}{2}\right)} 2^{\gamma} .
\end{aligned}
$$

Therefore $|\kappa(\pi)| \leq 1$ if and only if

$$
\nu+2 \mu_{2}+\frac{1}{2^{1-\gamma} \cos \left(\frac{\pi \gamma}{2}\right)} \mu_{\gamma} \leq 1
$$

Remark. Note that for $V=0$ and $d_{2}=0$ the stability condition for the resulting numerical method is

$$
\mu_{\gamma} \leq 2^{1-\gamma} \cos \left(\frac{\gamma \pi}{2}\right)
$$

Before proving the implicit numerical method is unconditionally stable we first present some results regarding the behavior of the coefficients $b_{m}$.

Lemma 4. The coefficient $b_{m}$ verifies:

(a) $\left|b_{m+1}\right| \leq\left|b_{m}\right|, \quad m \geq 1 ; \quad \lim _{m \rightarrow \infty} b_{m}=0 ;$

(b) $\sum_{m=-1}^{\infty} b_{m}=0$;

(c) $\sum_{m=-1}^{\infty} b_{m}(-1)^{m}=0$.

(d) $\sum_{m=-1}^{\infty} b_{m} \cos (m \theta) \geq 0$, for all $0 \leq \theta \leq \pi$.

The previous lemma can be easily proved. Note that, for $m \geq 2$ we have

$$
\begin{aligned}
b_{m}= & (m+2)^{2-\gamma}-2(m+1)^{2-\gamma}+2(m-1)^{2-\gamma} \\
& -(m-2)^{2-\gamma} .
\end{aligned}
$$

Therefore,

$$
\begin{aligned}
b_{m} & =m^{2-\gamma}\left[\left(1+\frac{2}{m}\right)^{2-\gamma}-2\left(1+\frac{1}{m}\right)^{2-\gamma}+2\left(1-\frac{1}{m}\right)^{2-\gamma}-\left(1-\frac{2}{m}\right)^{2-\gamma}\right] \\
& =m^{2-\gamma}\left[\sum_{k=0}^{\infty}\left(\begin{array}{c}
2-\gamma \\
k
\end{array}\right)\left(\frac{2}{m}\right)^{k}-2 \sum_{k=0}^{\infty}\left(\begin{array}{c}
2-\gamma \\
k
\end{array}\right)\left(\frac{1}{m}\right)^{k}+2 \sum_{k=0}^{\infty}\left(\begin{array}{c}
2-\gamma \\
k
\end{array}\right)\left(\frac{-1}{m}\right)^{k}-\sum_{k=0}^{\infty}\left(\begin{array}{c}
2-\gamma \\
k
\end{array}\right)\left(\frac{-2}{m}\right)^{k}\right] .
\end{aligned}
$$

Hence

$$
\begin{aligned}
b_{m} & =m^{2-\gamma}\left[\sum_{k=3}^{\infty}\left(\begin{array}{c}
2-\gamma \\
k
\end{array}\right)\left(\frac{2}{m}\right)^{k}-2 \sum_{k=3}^{\infty}\left(\begin{array}{c}
2-\gamma \\
k
\end{array}\right)\left(\frac{1}{m}\right)^{k}+2 \sum_{k=3}^{\infty}\left(\begin{array}{c}
2-\gamma \\
k
\end{array}\right)\left(\frac{-1}{m}\right)^{k}-\sum_{k=3}^{\infty}\left(\begin{array}{c}
2-\gamma \\
k
\end{array}\right)\left(\frac{-2}{m}\right)^{k}\right] \\
& =m^{2-\gamma}\left[(2-\gamma)(1-\gamma)(-\gamma) \frac{2}{m^{3}}+\cdots\right]=\frac{1}{m^{\gamma}}\left[(2-\gamma)(1-\gamma)(-\gamma) \frac{2}{m}+\cdots\right] .
\end{aligned}
$$

Considering (51), and by noting $k$ even terms of the series cancel, the properties (a) can be easily obtained. 
The condition (d) seems less straightforward. However, if we define the function $S(\theta)$ as

$S(\theta)=\sum_{m=-1}^{\infty} b_{m} \cos (m \theta), \quad$ for all $0 \leq \theta \leq \pi$,

we observe $S(\theta)$ is continuous in $[0, \pi]$ and it is only zero for $\theta=0$ and $\theta=\pi$ since we obtain respectively (b) and (c). By noting that for $\theta=\pi / 2$, the function $S$ is positive we can infer $S(\theta)$ is always positive. We plot in Fig. 3 the behavior of the function $S(\theta)$ that depends on $\gamma$ for $\gamma=0.3, \gamma=2 / 3$ and $\gamma=0.9$.

Theorem 5. The implicit numerical method (36) is unconditionally stable.

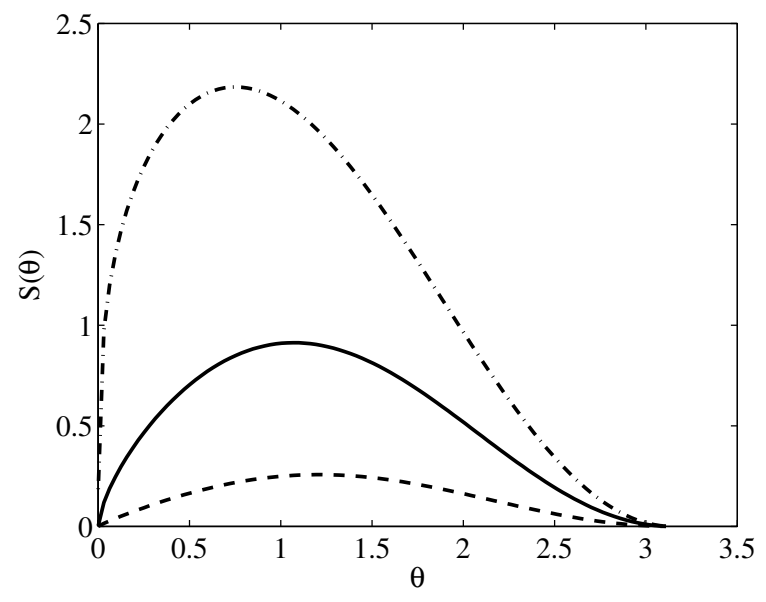

Fig. 3. Plot of the function $S(\theta)$ defined in (52) for $0 \leq \theta \leq$ $\pi$, and $\gamma=0.3(-\cdot), \gamma=2 / 3(-)$ and $\gamma=0.9(--)$.

Proof. If we insert the mode $\kappa^{n} \mathrm{e}^{i j \theta}$ into (36) then

$$
\begin{gathered}
\kappa(\theta)\left\{1-\frac{1}{2}\left[-\frac{\nu}{2}\left(\mathrm{e}^{i \theta}-\mathrm{e}^{-i \theta}\right)+\mu_{2}\left(\mathrm{e}^{i \theta}-2+\mathrm{e}^{-i \theta}\right)-\frac{\mu_{\gamma}}{2 \cos \left(\frac{\gamma \pi}{2}\right)}\left(\sum_{m=-1}^{\infty} b_{m} \mathrm{e}^{-i m \theta}+\sum_{m=-1}^{\infty} b_{m} \mathrm{e}^{i m \theta}\right)\right]\right\} \\
=1+\frac{1}{2}\left[-\frac{\nu}{2}\left(\mathrm{e}^{i \theta}-\mathrm{e}^{-i \theta}\right)+\mu_{2}\left(\mathrm{e}^{i \theta}-2+\mathrm{e}^{-i \theta}\right)-\frac{\mu_{\gamma}}{2 \cos \left(\frac{\gamma \pi}{2}\right)}\left(\sum_{m=-1}^{\infty} b_{m} \mathrm{e}^{-i m \theta}+\sum_{m=-1}^{\infty} b_{m} \mathrm{e}^{i m \theta}\right)\right] .
\end{gathered}
$$

If the real part of

$$
A=-\frac{\nu}{2}\left(\mathrm{e}^{-i \theta}-\mathrm{e}^{-i \theta}\right)+\mu_{2}\left(\mathrm{e}^{i \theta}-2+\mathrm{e}^{-i \theta}\right)-\frac{\mu_{\gamma}}{2 \cos \left(\frac{\gamma \pi}{2}\right)}\left(\sum_{m=-1}^{\infty} b_{m} \mathrm{e}^{-i m \theta}+\sum_{m=-1}^{\infty} b_{m} \mathrm{e}^{i m \theta}\right)
$$

is negative or zero then $|\kappa(\theta)| \leq 1$. Since

$$
\begin{array}{r}
A=-\nu \sin (\theta) i+2 \mu_{2}(\cos (\theta)-1) \\
-\frac{\mu_{\gamma}}{\cos \left(\frac{\gamma \pi}{2}\right)} \sum_{m=-1}^{\infty} b_{m} \cos (m \theta),
\end{array}
$$

from (52) we have $|\kappa(\theta)| \leq 1$.

\subsection{Numerical tests}

To test the numerical methods we need to have a problem for which we are able to compute an exact solution. Therefore, we start to consider an artificial problem, with no physical interpretation, since it is the easiest way to access an analytical solution. Hence, we consider a source term, that is, we consider the equation in the form

$$
\begin{aligned}
\frac{\partial u}{\partial t}(x, t)= & -V \frac{\partial u}{\partial x}(x, t)+d_{\gamma} \frac{\partial^{\gamma} u}{\partial|x|^{\gamma}}(x, t) \\
& +d_{2} \frac{\partial^{2} u}{\partial x^{2}}(x, t)+s(x, t),
\end{aligned}
$$

where $V, d_{\gamma}, d_{2}>0$ and in general we assume $0<\gamma<1$.

The problem is defined in the domain $[0,2]$ with an initial condition

$$
u(x, 0)=x^{4}(2-x)^{4}, \quad x \in[0,2]
$$

and boundary conditions

$$
u(0, t)=0 \quad \text { and } \quad u(2, t)=0 .
$$

This problem can be seen as defined in $\mathbb{R}$ by assuming $u(x, t)=0$ for $x<0$ and $x>2$. 
For the source term

$$
\begin{aligned}
s(x, t)= & \mathrm{e}^{-t}\left[-x^{2}(2-x)^{2}+8 V x^{3}(2-x)^{3}(1-x)\right. \\
& \left.+8 d_{2} x^{2}(2-x)^{2}\left(6(1-x)^{2}-x(2-x)\right)\right] \\
& +\frac{\mathrm{e}^{-t} d_{\gamma}}{2 \cos \left(\frac{\gamma \pi}{2}\right)} \sum_{p=0}^{4}(-1)^{p} 2^{4-p}\left(\begin{array}{l}
4 \\
p
\end{array}\right) \\
& \times \frac{\Gamma(p+5)}{\Gamma(p+5-\alpha)}\left(x^{p+4-\alpha}+(2-x)^{p+4-\alpha}\right),
\end{aligned}
$$

the exact solution, of the problem (53)-(55), is given by

$$
u(x, t)=\mathrm{e}^{-t} x^{4}(2-x)^{4} .
$$

To implement the finite difference schemes we consider the mesh points

$$
\begin{aligned}
& x_{j}=x_{0}+j \Delta x, \quad j=0,1,2, \ldots, N \quad \text { and } \\
& t_{n}=n \Delta t, \quad n \geq 0 .
\end{aligned}
$$

When implementing the numerical method we assume homogeneous boundary conditions

$$
U_{0}^{n}=U_{N}^{n}=0, \quad \text { for all } n .
$$

The numerical methods with source term are now respectively of the form

$$
\begin{aligned}
U_{j}^{n+1}= & U_{j}^{n}-\nu \Delta_{-} U_{j}^{n}+\mu_{\gamma} \Delta^{\gamma} U_{j}^{n} \\
& +\mu_{2} \delta^{2} U_{j}^{n}+s\left(x_{j}, t_{n}\right), \\
{\left[1-\frac{1}{2}\left(-\nu \Delta_{0}+\mu_{\gamma} \Delta_{2}^{\gamma}+\mu_{2} \delta^{2}\right)\right] U_{j}^{n+1} } & \\
= & {\left[1+\frac{1}{2}\left(-\nu \Delta_{0}+\mu_{\gamma} \Delta_{2}^{\gamma}+\mu_{2} \delta^{2}\right)\right] U_{j}^{n} } \\
& +\Delta t s_{j}^{n+1 / 2},
\end{aligned}
$$

where $s_{j}^{n+1 / 2}=s\left(x_{j}, t_{n+1 / 2}\right)$.

Table $1 . l_{\infty}$ error at $t=1$, for the explicit numerical method when $d_{\gamma}=1$ and $V=d_{2}=0$. We consider $\Delta t=0.01 \Delta x$ and for these values we have $\mu_{\gamma} \leq 0.008$ which is according to the stability condition (50).

\begin{tabular}{llll}
\hline$\Delta x$ & \multicolumn{1}{c}{$\gamma=0.3$} & \multicolumn{1}{c}{$\gamma=2 / 3$} & \multicolumn{1}{c}{$\gamma=0.9$} \\
\hline $2 / 50$ & $0.2940 \times 10^{-2}$ & $0.2280 \times 10^{-1}$ & $0.9145 \times 10^{-1}$ \\
$2 / 500$ & $0.3076 \times 10^{-3}$ & $0.2466 \times 10^{-2}$ & $0.1189 \times 10^{-1}$ \\
Rate & 0.98 & 0.97 & 0.89 \\
\hline
\end{tabular}

Table 2. $l_{\infty}$ error at $t=1$, for the implicit numerical method when $d_{\gamma}=1$ and $V=d_{2}=0$. We consider $\Delta t=\Delta x$. The numerical method is unconditionally stable.

\begin{tabular}{llll}
\hline$\Delta x$ & \multicolumn{1}{c}{$\gamma=0.3$} & \multicolumn{1}{c}{$\gamma=2 / 3$} & \multicolumn{1}{c}{$\gamma=0.9$} \\
\hline $2 / 50$ & $0.1332 \times 10^{-2}$ & $0.1431 \times 10^{-2}$ & $0.1417 \times 10^{-2}$ \\
$2 / 500$ & $0.1326 \times 10^{-4}$ & $0.1424 \times 10^{-4}$ & $0.1396 \times 10^{-4}$ \\
Rate & 2 & 2 & 2 \\
\hline
\end{tabular}

To show the convergence of the numerical methods, we consider the $l_{\infty}$ error, for an instant of time $t=n \Delta t$, given by

$$
\max _{j}\left|u\left(x_{j}, t\right)-U_{j}^{n}\right| .
$$

In the next tables we present the results concerning the convergence rate of both numerical methods: the first order explicit numerical method and the second order implicit numerical method. In Tables 1 and 2 we show the results with only the turbulent diffusion, that is, we consider Eq. (53) with $V=d_{2}=0$ and $d_{\gamma} \neq 0$, whereas in Tables 3 and 4 we consider additionally the advective term and the classical diffusion term. For the explicit numerical method we take into consideration the stability condition (49).

In Table 1, since the results concern the case for only turbulent diffusion, that is, for $\nu=\mu_{2}=0$, the stability condition is given by (50). In Table 2 we have the implicit numerical method which is unconditionally stable.

In Table 3 we need to take into consideration the stability condition (49), which involves $\nu, \mu_{2}, \mu_{\gamma}$. Although the stability condition was only proved to be a necessary condition, it is a very sharp condition. In Table 4 we present again the results for the implicit numerical method, which is unconditionally stable.

As expected the rate of convergence of the explicit numerical method in Tables 1 and 3 is one and the rate of convergence of the implicit numerical method in Tables 2 and 4 is two.

Table 3. $l_{\infty}$ error at $t=1$, for the explicit numerical method when $d_{\gamma}=1, V=0.25$ and $d_{2}=0.5$. We consider $\Delta t$ such that $\mu_{2}=0.01$ in order to have the stability condition (49), which involves $\nu, \mu_{2}, \mu_{\gamma}$.

\begin{tabular}{llll}
\hline$\Delta x$ & \multicolumn{1}{c}{$\gamma=0.3$} & \multicolumn{1}{c}{$\gamma=2 / 3$} & \multicolumn{1}{c}{$\gamma=0.9$} \\
\hline $2 / 50$ & $0.3324 \times 10^{-2}$ & $0.1078 \times 10^{-1}$ & $0.4702 \times 10^{-1}$ \\
$2 / 500$ & $0.3676 \times 10^{-3}$ & $0.1141 \times 10^{-2}$ & $0.5245 \times 10^{-2}$ \\
Rate & 0.96 & 0.98 & 0.95 \\
\hline
\end{tabular}


Table 4. $l_{\infty}$ error at $t=1$, for the implicit numerical method when $d_{\gamma}=1, V=0.25$ and $d_{2}=0.5$. We consider $\Delta t$ such that $\nu=0.1$. The numerical method is unconditionally stable.

\begin{tabular}{llll}
\hline$\Delta x$ & \multicolumn{1}{c}{$\gamma=0.3$} & \multicolumn{1}{c}{$\gamma=2 / 3$} & \multicolumn{1}{c}{$\gamma=0.9$} \\
\hline $2 / 50$ & $0.6326 \times 10^{-3}$ & $0.6999 \times 10^{-3}$ & $0.7507 \times 10^{-3}$ \\
$2 / 500$ & $0.6372 \times 10^{-5}$ & $0.7038 \times 10^{-5}$ & $0.7530 \times 10^{-5}$ \\
Rate & 1.99 & 1.99 & 1.99 \\
\hline
\end{tabular}

\section{Physical Behavior}

In this section we present the behavior of the solution for the problem which consists of Eq. (14) with the Gaussian initial condition

$$
u(x, 0)=\mathrm{e}^{-x^{2} / L^{2}},
$$

where $L$ is a scale parameter, and boundary conditions

$$
\lim _{|x| \rightarrow \infty} u(x, t)=0 .
$$

This initial profile is many times used to observe the physical behavior of problems involving diffusive processes, see for instance [Hotta et al., 2012].

The physical problem do not have boundaries and therefore since the computational domain needs to be bounded, we consider the computational domain large enough, so we can assume the numerical boundary conditions homogeneous. More specifically, we consider the domain in $x$ to be the interval $[-M, M]$ for $M$ large enough, that is, $M$ is such that the condition $u(x, t)=0$, for $|x|>M$, is a physical condition. In this way the numerical boundary

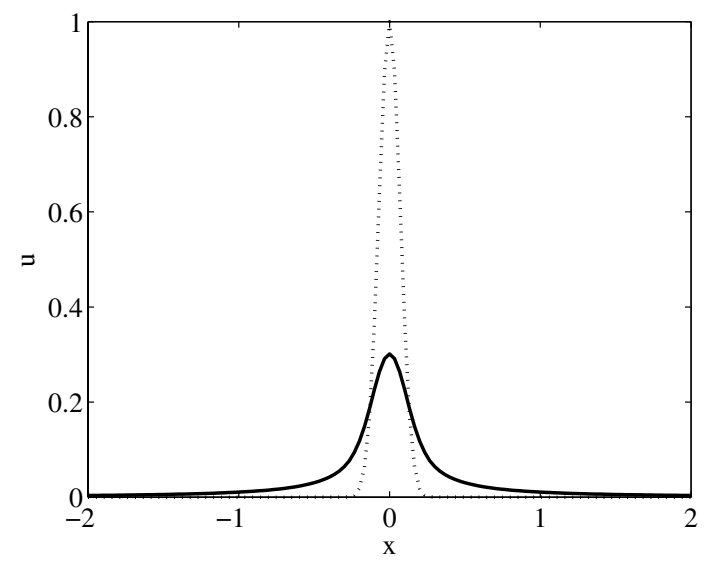

(a) conditions do not interfere with the accuracy of the numerical solution.

In Fig. 4, we display the behavior of the solution for the problem with only turbulent diffusion, that is, when we have $V=d_{2}=0$ and $d_{\gamma} \neq 0$, with $\gamma=2 / 3$. Figure 4(a) shows how the solution evolves from the instant $t=0$ until $t=0.3$. Figure 4(b) shows the differences between assuming different values of $d_{\gamma}$, namely $d_{\gamma}=0.5$ and $d_{\gamma}=1$.

In Fig. 5 we add some classical diffusion to the turbulent diffusion, that is, we consider $V=0$, but $d_{2} \neq 0$ and $d_{\gamma} \neq 0$. In Fig. 5(a), we show the effect of classical and turbulent diffusion separately and in Fig. 5(b) we show the effect when we have both diffusions.

In Fig. 6 we add the effect of the advective term. In Fig. 6(a), we show the behavior of the solution when $V$ increases and in Fig. 6(b) we show advection with turbulent diffusion and what happens with and without the advective term.

To end this section we also compare our equation with a super-diffusion equation, when subjected to the same initial and boundary conditions. We consider this problem since recently many applications have appeared where this type of diffusion is used as a model. We consider the equation

$$
\frac{\partial u}{\partial t}=d_{\alpha} \frac{\partial^{\alpha} u}{\partial|x|^{\alpha}}, \quad 1<\alpha<2
$$

where we define the Riesz derivative in a similar way to what we have done for $0<\gamma<1$ in (12), but now for $1<\alpha<2$. The numerical solution

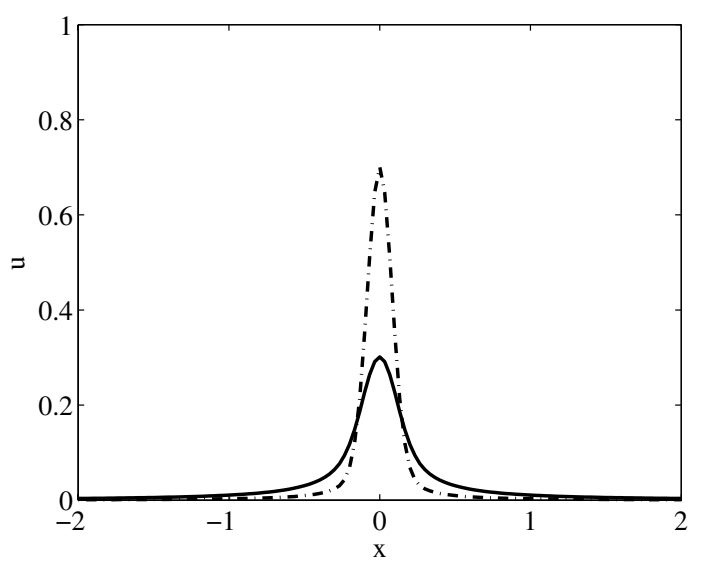

(b)

Fig. 4. Solutions for the turbulent diffusion problem, that is, when $V=0, d_{2}=0$ and $d_{\gamma} \neq 0$. The solutions are plotted at the instant $t=0.3$ and for $\gamma=2 / 3$ : (a) Initial condition for $L=0.1(\cdots)$ and solution for $d_{\gamma}=1(-)$ and (b) solution for $d_{\gamma}=0.5(-\cdot)$ and solution for $d_{\gamma}=1(-)$. 


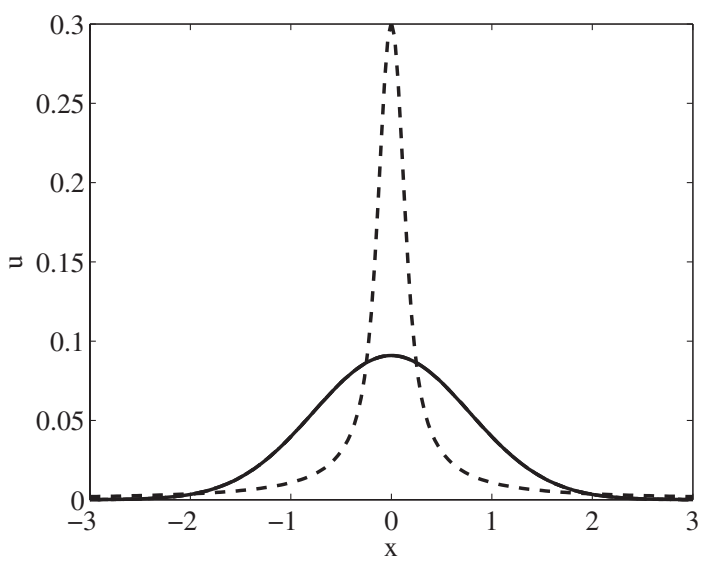

(a)

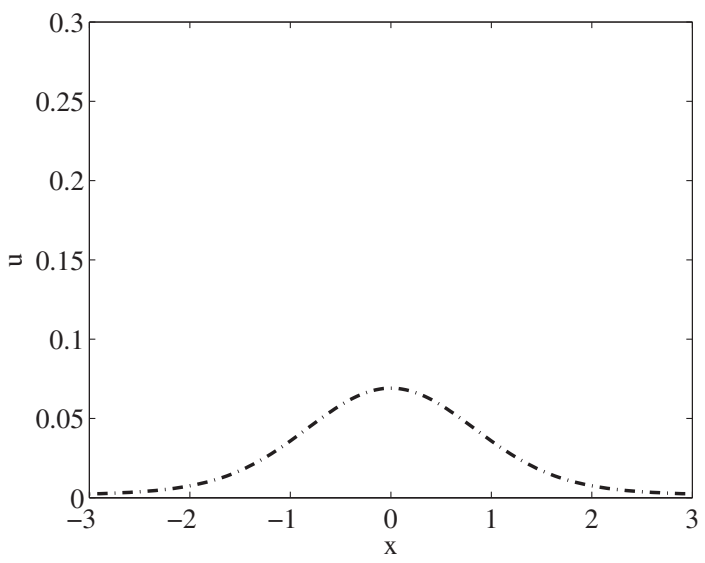

(b)

Fig. 5. Solutions with no advective term, that is, for $V=0$. The solutions are plotted at the instant $t=0.3$ and for $\gamma=2 / 3$ : (a) Solution for $d_{\gamma}=0, d_{2}=1(--)$ and solution for $d_{\gamma}=1, d_{2}=0(-)$ and (b) solution for $d_{\gamma}=1, d_{2}=1(-\cdot)$.

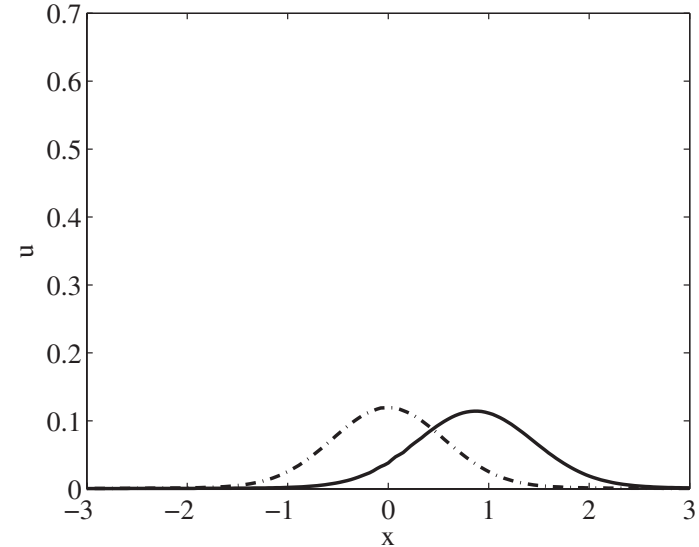

(a)

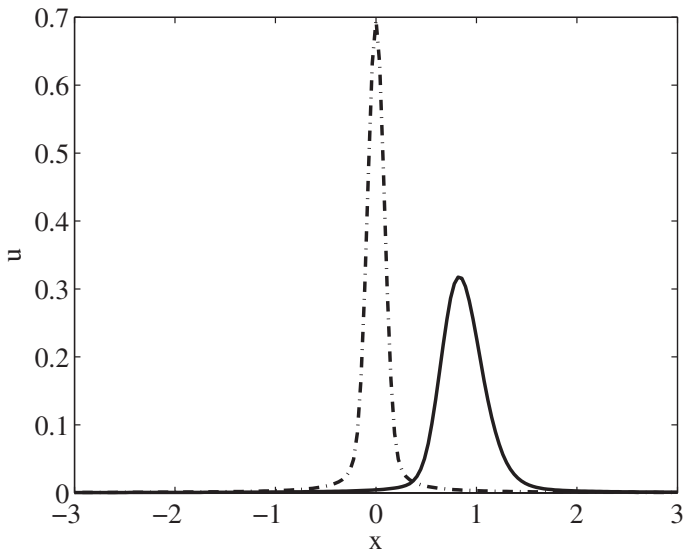

(b)

Fig. 6. Solutions with and without advection at the instant $t=0.3$ and for $\gamma=2 / 3$ : (a) Solution for $V=0, d_{\gamma}=0.5$, $d_{2}=0.5(-\cdot)$ and solution for $V=3, d_{\gamma}=0.5, d_{2}=0.5(-)$ and (b) solution with only turbulent diffusion, that is, $d_{2}=0$ : for $V=0, d_{\gamma}=0.5(-\cdot)$ and for $V=3, d_{\gamma}=0.5(-)$.

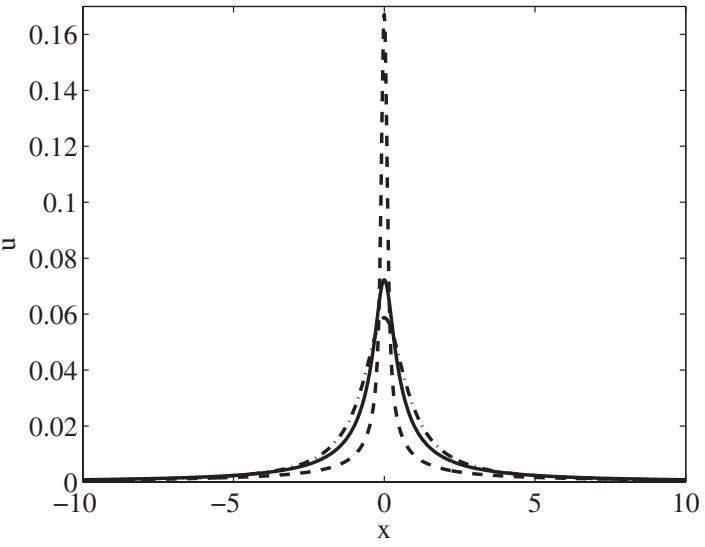

(a)

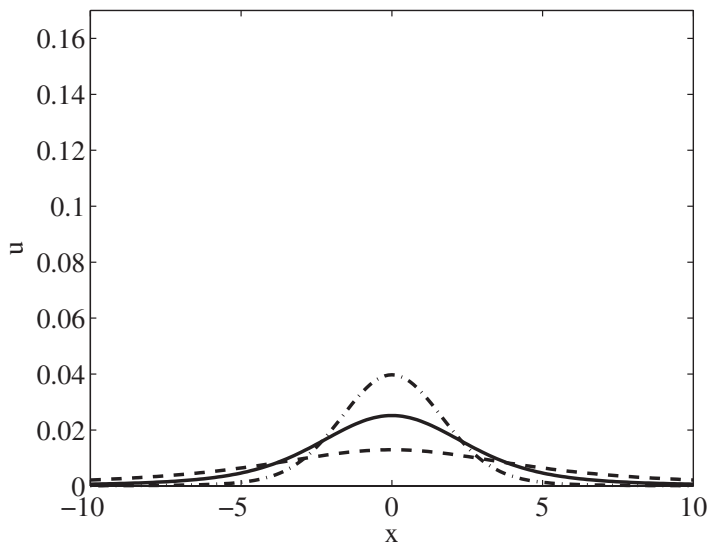

(b)

Fig. 7. Turbulent diffusion versus super-diffusion at $t=1$. (a) Turbulent diffusion: solution for $V=d_{2}=0$ and $d_{\gamma}=1$ with $\gamma$ changing $\gamma=0.3(---), 2 / 3(-), 0.9(--)$ and (b) super-diffusion: solution for $d_{\alpha}=1$ with $\alpha$ changing $\alpha=1.2$ (---), 1.5 $(-), 1.8(-\cdot)$. 


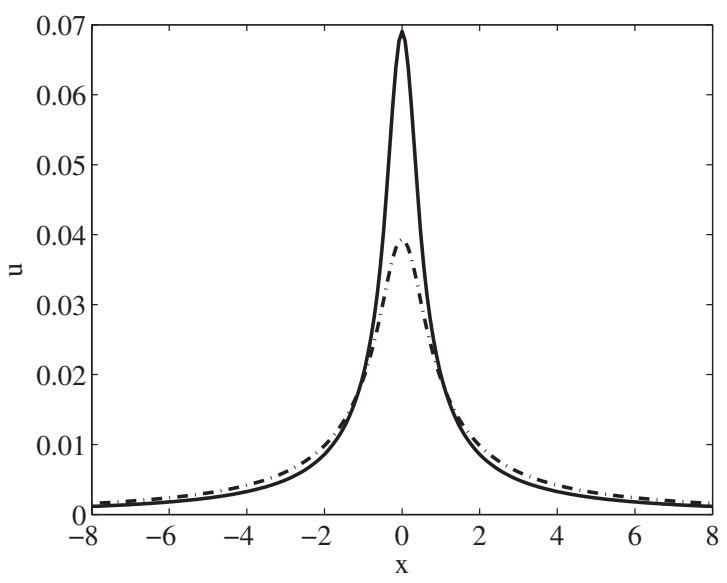

(a)

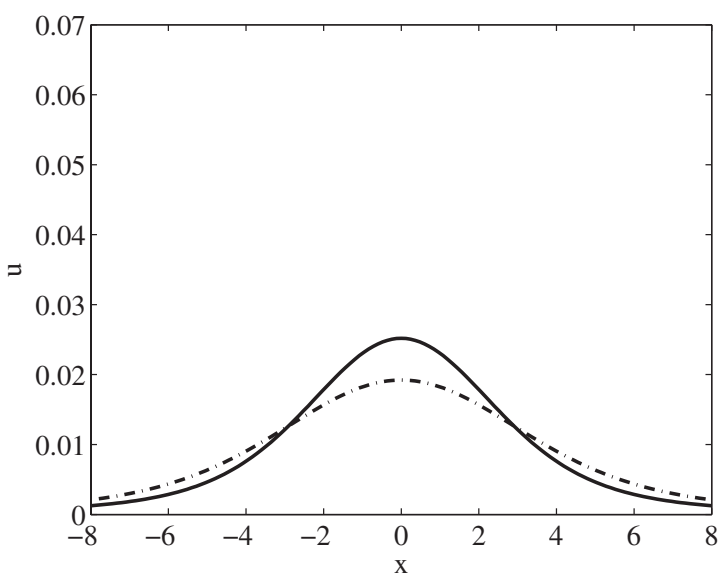

(b)

Fig. 8. Turbulent diffusion versus super-diffusion at $t=1(-)$ and $t=1.5$ (---). (a) Turbulent diffusion: solution of (13) for $V=d_{2}=0$ and $d_{\gamma}=1$ for $\gamma=2 / 3$ and (b) super-diffusion: solution of (59) for $\alpha=1.5$.

of this equation is computed using the numerical discretization presented in [Sousa, 2012a].

In Figs. 7 and 8 we plot separately superdiffusion and turbulent diffusion. In Fig. 7 we plot the solution at instant $t=1$ and for different values of $\gamma[7(\mathrm{a})]$ and $\alpha[7(\mathrm{~b})]$ and in Fig. 8 we consider $\gamma=2 / 3[8(\mathrm{a})]$ and $\alpha=1.5[8(\mathrm{~b})]$ at different instants of time. We observe the profiles are quite different and the diffusive processes affect the same regions differently, that is, considerably higher values of $u$ concentrate in some regions for the turbulent case. In practical applications, this can mean that if we disregard the turbulent diffusion, the model may assume wrongly that for a certain time the concentration of $u$ will disappear in certain regions.

\section{Final Remarks}

We have considered a model which includes advection, classical diffusion and turbulent diffusion. Two numerical methods of first and second orders respectively have been derived and convergence results have been given. The numerical tests confirm that the numerical solution converges to the exact solution, presenting the order of accuracy discussed analytically and also the stability conditions have shown to be very sharp. In the last section we have shown how the turbulent diffusion evolves if we have a Gaussian initial condition. We have also displayed side by side the phenomenon of super-diffusion to give a general overview of different phenomena, such as, turbulent diffusion, classical diffusion and super-diffusion.

\section{Acknowledgment}

Research supported by CMUC and FCT (Portugal), through European program COMPETE/FEDER.

\section{References}

Bakunin, O. G. [2004] "Correlation effects and turbulent diffusion scalings," Rep. Prog. Phys. 67, 965-1032.

Bakunin, O. G. [2008] Turbulence and Diffusion (Springer).

Boffetta, G. \& Sokolov, I. M. [2002] "Relative dispersion in fully developed turbulence: The Richardson's law and intermittency corrections," Phys. Rev. Lett. 88, 094501.

Chen, L. \& Deem, M. [2001] "Reaction, Lévy flights and quenched disorder," Phys. Rev. E 65, 011109.

Chen, W. [2006] "A speculative study of $2 / 3$-order fractional Laplacian modeling of turbulence: Some thoughts and conjectures," Chaos 16, 023126.

Chukbar, K. V. [1993] "Toward the theory of turbulent diffusion," Pis'ma Zh. Eksp. Teor. Fiz. 58, 87-90.

Cushman-Roisin, B. [2008] "Beyond eddy diffusivity: An alternative model for turbulent dispersion," Environ. Fluid Mech. 8, 543-549.

del-Castillo-Negrete, D., Carreras, B. A. \& Lynch, V. E. [2005] "Nondiffusive transport in plasma turbulence: A fractional diffusion approach," Phys. Rev. Lett. 94, 065003.

Diethelm, K., Ford, N. J. \& Freed, A. D. [2004] "Detailed error analysis for a fractional Adams method," Numer. Algorithms 36, 31-52.

Hotta, H., Iida, Y. \& Yokoyama, T. [2012] "Estimation of turbulent diffusivity with direct numerical simulation of stellar convection," The Astrophys. J. Lett. 751, L9. 
Li, C., Chen, A. \& Ye, J. [2011] "Numerical approaches to fractional calculus and fractional ordinary differential equation," J. Comput. Phys. 230, 3352-3368.

Li, C. \& Zeng, F. [2012] "Finite difference methods for fractional differential equations," Int. J. Bifurcation and Chaos 22, 1230014.

Lubich, Ch. [1986] "Disctretized fractional calculus," SIAM J. Math. Anal. 17, 704-719.

Majda, A. J. \& Kramer, P. R. [1999] "Simplified models for turbulent diffusion: Theory, numerical modelling and physical phenomena," Phys. Rep. 314, 237-574.

Ortigueira, M. [2006] "Riesz potential operators and inverses via fractional centred derivatives," Int. J. Math. Sci. 2006, 48391.

Podlubny, I. [1999] Fractional Differential Equations (Academic Press, San Diego).

Porta, A. L., Voth, G. A., Crawford, A. M., Alexander, J. \& Bodenschatz, E. [2001] "Fluid particle accelerations in fully developed turbulence," Nature $\mathbf{4 0 9}$, 1017-1019.

Roberts, P. J. W. \& Webster, D. R. [2002] "Turbulent diffusion," Conf. Proc. Environmental Fluid
Mechanics: Theories and Applications (American Society of Civil Engineers), pp. 7-45.

Samko, S. G., Kilbas, A. A. \& Marichev, O. I. [1993] Fractional Integrals and Derivatives: Theory and Applications (Gordon and Breach Science Publishers).

Shlesinger, M. F., West, B. J. \& Klafter, J. [1987] "Lévy dynamics of enhanced diffusion: Application to turbulence," Phys. Rev. Lett. 58, 1100-1103.

Shlesinger, M. F., Zaslavsky, G. M. \& Klafter, J. [1993] "Strange kinetics," Nature 363, 31-37.

Sousa, E. [2012a] "A second order explicit finite difference method for the fractional advection diffusion equation," Comp. Math. Appl. 64, 3141-3152.

Sousa, E. [2012b] "How to approximate the fractional derivative of order $1<\alpha \leq 2$," Int. J. Bifurcation and Chaos 22, 1250075.

Sreenivasan, K. R. \& Antonia, R. A. [1997] "The phenomenology of small-scale turbulence," Ann. Rev. Fluid Mech. 29, 435-472.

Zaslavsky, G. M. [2002] "Chaos, fractional kinetics, and anomalous transport," Phys. Rep. 371, 461-580. 